\title{
CALIDAD DE VIDA RELACIONADA CON LA SALUD EN MUJERES URUGUAYAS CON CÁNCER DE MAMA
}

\section{HEALTH RELATED QUALITY OF LIFE OF URUGUAYAN WOMEN WITH BREAST CANCER}

\author{
Laura Bajuk \\ Universidad Católica del Uruguay, Uruguay \\ Micaela Reich \\ Universidad Católica del Uruguay, Uruguay
}

\begin{abstract}
Resumen: Existen diversas investigaciones científicas que estudian la relación entre Cáncer de Mama y Calidad de Vida. Sin embargo, la investigación en Uruguay es aún incipiente. Este estudio tiene como objetivo principal la caracterización de una muestra de mujeres uruguayas con Cáncer de Mama recibiendo atención médica post-quirúrgica en el Instituto Nacional de Cáncer. La muestra analizada (n=34 mujeres) completó un formulario de Datos Sociodemográficos y Clínicos diseñado ad hoc para este estudio y el Cuestionario de Salud MOS-SF-36 V. 2.0. La media de edad de las participantes fue de 56,06 años. La mayoría de las participantes recibió Quimioterapia (73,5\%), Radioterapia (64,7\%) o la combinación de ambos (58,8\%). El tipo de cirugía más frecuente fue la Mastectomía Radical Modificada (53\%). Se hallaron correlaciones estadísticamente significativas entre variables clínicas, sociodemográficas y el SF-36. Se concluyó que el tipo de cirugía así como el tratamiento recibido tienen una importante incidencia en la Calidad de Vida Relacionada con la Salud de estas mujeres.
\end{abstract}

Palabras clave: cáncer, cáncer de mama, calidad de vida, calidad de vida relacionada con la salud, Psicooncología.

\begin{abstract}
There is an extensive body of scientific literature that studies the relationship between Breast Cancer and Quality of Life. However, studies in Uruguay are still emerging. The aim of this study was to characterize a sample of Uruguayan women with Breast Cancer receiving post-surgical attention at the National Cancer Institute. Thirty-four women completed a Clinical and Socio-demographic form designed ad hoc for this investigation. Health Related Quality of Life was assessed by the MOS-SF-36 V. 2.0. questionnaire. The sample's mean age was 56,06 years. Most women had received Chemotherapy (73,5\%), Radiotherapy (64,7\%) or the combination of both (58,8\%). Fifty-three percent had undergone Modified Radical Mastectomy. Significant correlations emerged between clinical and socio-demographic variables and SF-36 subscales. Our findings suggest that the type of surgery and adjuvant treatment undergone may have significant impact on these women's Health Related Quality of Life.
\end{abstract}

Keywords: cancer, breast cancer, quality of life, health related quality of life, Psycho-oncology.

\section{INTRODUCCIÓN}

Actualmente, la Calidad de Vida del enfermo oncológico se considera una de las principales áreas de investigación de la Psicooncología (Remor, Ulla, y Arranz, 2003); resultando esencial para identificar aquellos pacientes que necesiten de una intervención psicosocial (Bardwell et al., 2004). "El Cáncer de Mama es el tumor que más atención ha recibido desde la Psicología, y en el que se han realizado un número importante de estudios de Calidad de Vida" (Arraras et al., 2000, p.334).
Datos epidemiológicos nacionales señalan que "en el Uruguay el Cáncer de Mama es el de mayor incidencia en la mujer uruguaya y constituye la primera causa de muerte por cáncer en la mujer" (Vasallo y Barrios, 2003, p.108).

Conceptualizamos al Cáncer de Mama como (a) una enfermedad grave que sigue un curso a nivel de aspectos clínicos tal, que es susceptible de verse afectada por múltiples factores comportamentales y biológicos y (b) un estresor crónico que genera en el individuo que lo padece diversas limitaciones a nivel psicológico y físico- limitaciones que podrían desbordar sus recursos de afrontamiento (Antoni, 2002).

Correspondencia: Micaela Reich. Facultad de Psicología, Universidad Católica del Uruguay.

Correo Electrónico: mreich@ucu.edu.uy. 
Se define a la Calidad de Vida Relacionada con la Salud como un concepto subjetivo y multidimensional (Bullinger, 1991). De acuerdo con Schwartzmann (2003), la Calidad de Vida es una noción eminentemente humana que se relaciona con el grado de satisfacción que la persona tiene sobre su condición física, su estado emocional, su vida familiar, amorosa y social, así como con el sentido que le otorga a su propia vida.

La Calidad de Vida de las pacientes con Cáncer de Mama puede verse influenciada tanto por variables de tipo biológico o físico, como ser el estadío de la enfermedad, el tipo de tratamiento y sus efectos secundarios, el pronóstico y las posibilidades de rehabilitación; como por variables psicosociales como la imagen corporal, el status emocional y las relaciones de pareja (Arraras, Illarramendi, Manterola, Salgado, y Domínguez, 2007). Podemos afirmar que el constructo de Calidad de Vida Relacionada con la Salud (en adelante CVRS) está orientado hacia la perspectiva de la persona afectada, considerando a ésta como el mejor evaluador de su propia Calidad de Vida (Aaronson 1993; Barreto y Bayés, 1990; Fossa, 1996; Gaite, 2008; Hietanen, 1996; Osoba, 1994).

En relación a los tratamientos, existen numerosas investigaciones que han concluido que el tratamiento médico adyuvante relacionado con una más marcada disminución en la Calidad de Vida del paciente oncológico es la Quimioterapia (Hopwood, Haviland, Mills, Sumo, y Bliss, 2007; Meyerowitz, Sparks, y Spears, 1978). Sin embargo, se deberá tomar en cuenta que la eficacia de los tratamientos se mide no sólo en términos del período de remisión de la enfermedad y del tiempo de supervivencia, sino también en términos de la Calidad de Vida de la persona enferma, su funcionamiento social y familiar, y el grado de ausencia de malestar físico y psicológico (Barroilhet, Forjaz y Garrido, 2005). Se debe tener presente que todas las opciones de tratamiento afectan, de una forma u otra, la CVRS de estos pacientes (Radice y Redaelli, 2003). Sin embargo, Montazeri et al. (2009) argumentan que la mayoría de los aspectos de la CVRS mejorarán una vez finalizado el tratamiento adyuvante; y que los efectos residuales no perdurarán en la mayoría de las pacientes.

El abordaje integral en el tratamiento de enfermedades crónicas como el Cáncer de Mama resulta ineludible, dado que no siempre las personas con mayor compromiso orgánico y/o con mayor sintomatología física de la enfermedad y sus tratamientos son las que presentan mayores dificultades a nivel emocional, psicológico y social. Es decir que si bien la Calidad de Vida del paciente oncológico está determinada por el grado de afectación física de la enfermedad, no siempre se observa una relación directa entre la gravedad de su sintomatología y el deterioro de su Calidad de Vida.

El tipo de cirugía recibida ha sido ampliamente documentado como factor asociado a la CVRS. Se ha encontrado que tiene un mayor impacto en detrimento de ésta la Mastectomía frente a la Cirugía Conservadora (de Haes, Curran, Aaronson, y Fentiman, 2003; Rabin, Heldt, Hirakata, y Fleck, 2008; Wapnir, Cody, y Greco, 1999). Además, se encontró que la Mastectomía conlleva un impacto negativo en la imagen corporal de la mujer con Cáncer de Mama (Arraras, Martínez, Manterota, y Laínez, 2004; Janni et al. 2001), por lo que la Cirugía Conservadora es el tratamiento que menos afecta la Calidad de Vida, incluso en pacientes por encima de los 70 años (de Haes et al.; Schou, Ekeberg, Sandvik, Hjermstad, y Ruland, 2005). Estos resultados son coincidentes con los arrojados previamente por el estudio de Curran et al. (1998).

En relación a las variables sociodemográficas y clínicas asociadas a la Calidad de Vida, se ha encontrado que bajos niveles de educación (National Breast Cancer Center [NBCC], 2009; Schou et al., 2005), bajos ingresos (NBCC), mayor severidad de la enfermedad, tratamientos adyuvantes, el hecho de vivir solos (Schou et al.), joven edad (Radice y Redaelli, 2003; Schou et al.; Shapiro et al., 2001) e incidencia de metástasis (Lidgren, Wilking, Jonsson, y Rehnberg, 2007) constituyen factores que afectan negativamente la Calidad de Vida de las mujeres. Asimismo, ha sido documentado que las mujeres laboralmente activas presentan mejores niveles de Calidad de Vida (Safee, Moghimi-Dehkordi, Zeighami, Tabatabaee, y Pourhoseingholi, 2008). En lo que respecta a la influencia de estar en pareja al momento de afrontar el Cáncer de Mama, se encontró que el sostén de la pareja es uno de los aspectos de mayor importancia en la CVRS. A raíz de esto, puede afirmarse que es la calidad de la relación de la pareja lo que influye en la Calidad de Vida (Shapiro et al.). De acuerdo con el NBCC, el hecho de no estar involucrado en una relación de pareja es considerado un factor que contribuye al 
distress, afectando su bienestar psicosocial; esto aumentaría la vulnerabilidad de las mujeres con Cáncer de Mama.

El presente estudio pretende arrojar luz sobre los aspectos clínicos, sociales y demográficos asociados a diferentes niveles de Calidad de Vida, buscando conocer las características de la población uruguaya en función de la literatura revisada que evalúa este constructo desde una metodología de autoinforme.

\section{MÉTODO}

\section{Participantes}

La muestra de participantes estuvo compuesta por mujeres uruguayas con Cáncer de Mama post-cirugía $(n=34)$, que se encontraran próximas al período de inicio, durante, o bien habiendo finalizado los tratamientos coadyuvantes (Radioterapia y/o Quimioterapia, por ejemplo) en diferentes estadíos de extensión de la enfermedad (I, II, III y IV), de acuerdo a clasificación clínica del Cáncer de Mama $(\mathrm{NCl}$, 2009) y estuvieran recibiendo asistencia en el Instituto Nacional de Cáncer (INCA).

Fueron considerados criterios de inclusión: (a) que haya transcurrido más de un mes desde el momento del diagnóstico; (b) haber tenido su primera cirugía para el tratamiento de Cáncer de Mama; (c) participación voluntaria y anónima; (d) nivel cultural que habilite a la comprensión de los cuestionarios; (e) estar recibiendo asistencia y acudiendo a consulta externa en el INCA.

Fueron considerados criterios de exclusión: (a) estar consumiendo psicofármacos; (b) presentar cualquier diagnóstico de desorden mental o cognitivo que pudiera dificultar la comprensión de los cuestionarios; (c) presentar otras enfermedades crónicas.

\section{Instrumentos}

a) MOS-SF-36 V. 2.0. para Uruguay (Ware, 2000). Este cuestionario evalúa la Calidad de Vida en poblaciones adultas (mayores de 16 años). El producto de su aplicación es la construcción de 8 dimensiones o subescalas, resultado del promedio de la suma de las preguntas contenidas en el cuestionario. Estas subescalas son: Función Física (FF), Rol Físico (RF), Dolor Corporal (DC), Salud General (SG), Vitalidad (VT), Función Social
(FS), Rol Emocional (RE) y Salud Mental (SM). Además, estas 8 subescalas pueden ser agrupadas en un Componente de Salud Físico y un Componente de Salud Mental. El SF-36 incluye también el concepto general de cambios en la percepción del estado de salud actual respecto del año anterior. La respuesta a esta pregunta describe la transición de la percepción respecto a la mejora o empeoramiento del estado de salud. La fiabilidad de las subescalas ha estado comprendida entre .81 y .88 en amplias muestras comunitarias de adultos (p.ej.,Stewart et al., 1989; Stewart, Hays, y Ware, 1988). El SF-36 es un instrumento de autoinforme, contiene 36 preguntas con un sistema de respuesta tipo Likert, que varía en algunos ítems de la escala y un ítem (ítem 2) que hace referencia a la Evolución Declarada de la Salud (HT). Toma un promedio de 8 a 12 minutos para ser cumplimentado. Por cada escala, las respuestas a cada pregunta se codifican y recodifican (10 preguntas), y los resultados se trasladan a una escala de 0 (peor salud) a 100 (mejor salud) (Alonso, 1999). La puntuación de cada dimensión fue calculada sumando las respuestas después de invertir algunos de ellos para asegurar que siempre una puntuación mayor indicase mejor Calidad de Vida. El instrumento tiene un alto grado de aceptabilidad y la calidad de los datos proporcionados es muy buena (Alonso). Los autores originales de este cuestionario son Ware y Sherbourne (1992), del Health Institute New England Medical Center de Boston Massachussets.

b) Formulario de Datos Sociodemográficos y Clínicos. El formulario incluyó: tiempo transcurrido desde el diagnóstico; fase clínica; tratamientos recibidos por el diagnóstico de Cáncer al momento de la evaluación; diagnósticos anteriores de Cáncer; antecedentes familiares de Cáncer; nivel de información sobre el diagnóstico y los tratamientos; estadío de la enfermedad; fuente de información sobre la propia salud; tiempo de internación a lo largo del último año; presencia de otras enfermedades crónicas; consulta con psicólogo u otro profesional de la salud y motivo de la misma; diagnóstico de trastorno de salud mental; tratamiento psicológico o psiquiátrico. Asimismo, se solicitó información referida: edad; estado civil; convivencia con otras personas; existencia de hijos y convivencia con los mismos; edad del hijo menor e hijo mayor; creencia religiosa; lugar de residencia; nivel de estudios formales alcanzado; profesión y status laboral. 


\section{Procedimientos}

Una vez obtenida la aprobación del Comité de Ética en Investigación con Seres Humanos de la Universidad Católica del Uruguay, se llevaron adelante diversas entrevistas con Directivos del INCA, y habiendo obtenido con sentimiento por parte de éstos, así como del cuerpo médico responsable del Departamento de Oncología Clínica de la Institución, se reclutó a las participantes en el momento en que salieran de consulta con su médico tratante (previa revisión de Historia Clínica). Se les explicaron los aspectos relevantes del estudio y se obtuvo el consentimiento informado. Una vez las mujeres manifestaron por escrito su participación voluntaria, se realizó una única evaluación por medio de la cumplimentación de cuestionarios. Las pacientes fueron asistidas en dicho proceso por profesionales o estudiantes avanzados integrantes del equipo de investigación.
El tiempo aproximado de evaluación por participante fue de entre 15 y 20 minutos.

\section{Diseño}

Se condujo un estudio epidemiológico de tipo descriptivo, comparativo, correlacional, y transversal.

Los datos fueron analizados por medio del paquete estadístico SPSS versión 18.0 para Windows.

\section{RESULTADOS}

Se realizó una caracterización de la muestra de mujeres uruguayas con Cáncer de Mama de acuerdo a variables sociodemográficas, tipo de tratamiento, y tipo de cirugía recibidos.

La Tabla 1 resume las características sociodemográficas de la muestra.

Tabla 1

Variables sociodemográficas

\begin{tabular}{lclc}
\hline Variables sociodemográficas & $\%$ & \multicolumn{1}{c}{ Variables sociodemográficas } & $\%$ \\
\hline Edad (Media: 56,06 ) & 32,4 & Religión & 94,1 \\
Grupo 38 a 49 años & 35,5 & No creyente & 5,9 \\
Grupo 50 a 59 años & 32,4 & Antecedentes familiares de Cáncer & 79,4 \\
Grupo 60 a 78 años & & Si & 20,6 \\
Estado civil & 41,2 & No & 55,9 \\
Casada & 23,5 & Profesión & 17,6 \\
Viuda & 20,6 & Ama de casa & 17,6 \\
Divorciada & 11,8 & Funcionaria & 8,8 \\
Soltera & 2,9 & Trabaja por cuenta propia & \\
Pareja de hecho & & Trabaja para otro & 5,9 \\
Personas con las que vive & 26,5 & Estudios formales & 50,0 \\
Vive sola & 23,5 & Sin estudios & 38,2 \\
Con hijos & 23,5 & Primaria completa \\
En pareja y con hijos & 17,6 & Secundaria completa & 5,9 \\
En pareja & 5,9 & Bachillerato u otros estudios técnicos & \\
Con hermanos & 2,9 & Dónde vive & 73,5 \\
Con amigos & & Montevideo & 20,6 \\
Situación laboral actual & 23,5 & Canelones & 5,8 \\
Activa & 11,8 & Otros departamentos \\
Incapacidad laboral transitoria & 8,8 & Tiene hijos & 88,2 \\
Incapacidad laboral permanente & 26,5 & Si & 11,8 \\
Jubiladas & 26,5 & No & \\
Pensionistas & 2,9 & & \\
Otros & & & \\
\hline
\end{tabular}


En la Tabla 2 se presenta la caracterización de la muestra según el Tipo de Tratamiento recibido.

El tipo de tratamiento más frecuentemente recibido fue la Quimioterapia en primer lugar $(73,5 \%)$, seguido por la Radioterapia $(64,7 \%)$ y la combinación de ambos (58,8\%).

En la Tabla 3 puede verse la muestra de mujeres agrupadas según el Tipo de Cirugía recibida. El tipo de cirugía más frecuentemente recibido fue, en primer lugar, la Mastectomía
Radical Modificada (53\%), seguido por la Cirugía Conservadora (47\%).

En los siguientes gráficos se presenta el número de participantes agrupadas según las puntuaciones obtenidas en los Componentes de Salud Físico y Salud Mental del MOS-SF-36 V. 2.0. La mayoría de las participantes alcanzaron puntuaciones en el Componente de Salud Físico entre 71 y 80 , valores relativamente altos de funcionamiento físico. No se registraron valores reportados por debajo de 30 .

Tabla 2

Tipo de tratamiento adyuvante recibido

\begin{tabular}{lcc}
\hline Tratamiento adyuvante recibido & $n$ & $\%$ \\
\hline Quimioterapia & 25 & 73,5 \\
Recibieron & 9 & 26,5 \\
No recibieron & 22 & 64,7 \\
Radioterapia & 12 & 35,3 \\
Recibieron & & \\
$\quad$ No recibieron & 20 & 38,2 \\
Hormonoterapia & 13 & 58,8 \\
Recibieron & & \\
No recibieron & 2 & 5,9 \\
Inmunoterapia & 31 & 91,2 \\
Recibieron & & 58,8 \\
No recibieron & 20 & 41,2 \\
Combinación de Quimioterapia y Radioterapia & 14 & \\
Recibieron & & \\
No recibieron & & \\
\hline
\end{tabular}

Tabla 3

Tipo de cirugía recibida

\begin{tabular}{lll}
\hline Cirugia recibida & $n$ & $\%$ \\
\hline Cirugía Conservadora & 16 & 47 \\
Mastectomía radical Modificada & 18 & 53
\end{tabular}

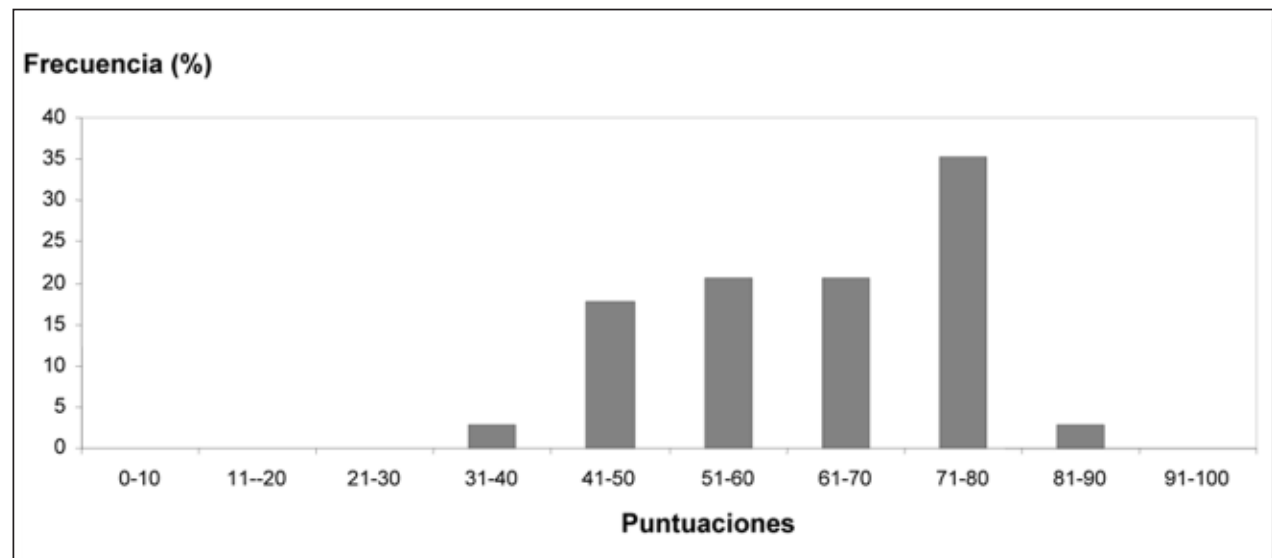

Figura 1. Componente de Salud Física. 


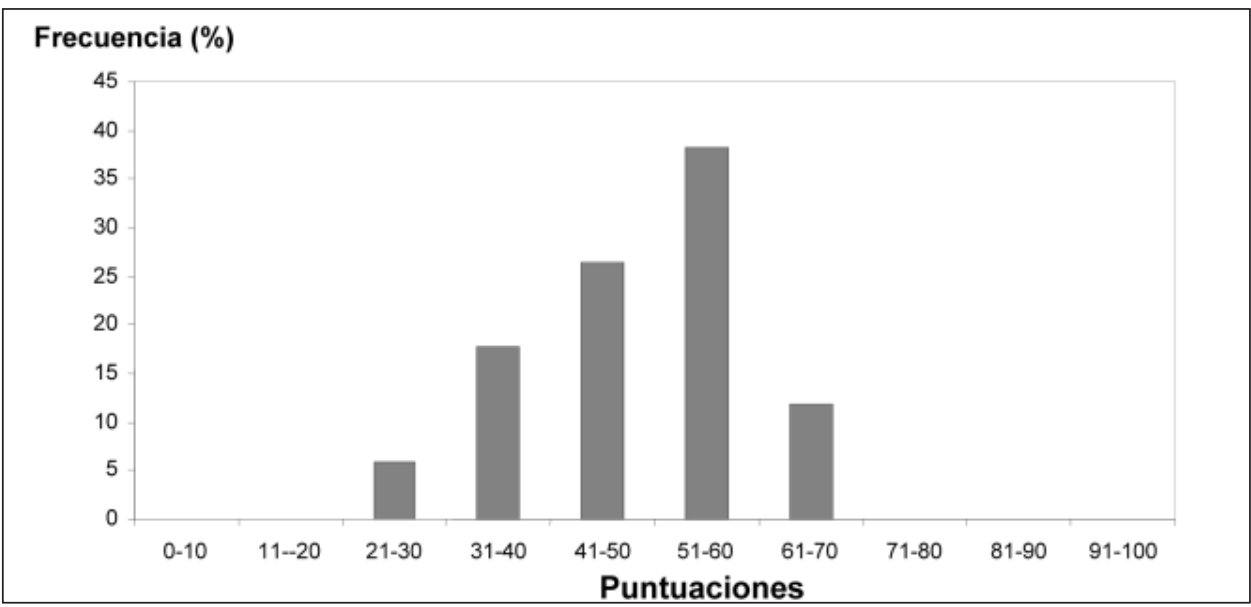

Figura 2. Componente de Salud Mental.

El rango de valores del Componente de Salud Mental de las participantes se encontró desplazado hacia valores inferiores en la escala, asociados éstos con peores niveles de salud mental.
Se condujo un análisis correlacional entre las variables de estudio y la CVRS. En la Tabla 4 se presentan las correlaciones entre las variables clínicas y sociodemográficas con las escalas del SF-36.

Tabla 4

Correlaciones entre variables clínicas y sociodemográficas con las subescalas del MOS-SF-36 V. 2.0

MOS-SF-36 V. 2.0.

\begin{tabular}{|c|c|c|c|c|c|c|c|c|c|}
\hline Variables & $F F$ & $R F$ & $D C$ & $S G$ & $V$ & FS & $R E$ & $S M$ & $p$ \\
\hline Estadio de la enfermedad & -.109 & -.189 & -.085 & .109 & -.050 & -.041 & -.229 & .133 & $p \leq .05$ \\
\hline Fase clínica actual & -.374 & .299 & .142 & .035 & .002 & .163 & .355 & .113 & $\mathrm{p} \leq .05$ \\
\hline Situación laboral & -.486 & -.023 & -.128 & .101 & -.063 & .339 & .110 & .012 & $\mathrm{p} \leq .05$ \\
\hline Tipo de Cirugía & -.038 & -.160 & .183 & .187 & .341 & .091 & .095 & .364 & $\mathrm{p} \leq .05$ \\
\hline Radioterapia & .074 & .088 & -.378 & -.134 & .177 & .081 & .190 & .256 & $\mathrm{p} \leq .05$ \\
\hline Quimioterapia & -0.37 & .027 & .107 & -.409 & -.135 & -.009 & .050 & -0.89 & $p \leq .05$ \\
\hline Inmunoterapia & -.041 & -.029 & .061 & .067 & .089 & -.087 & -.205 & -.033 & $\mathrm{p} \leq .05$ \\
\hline Hormooterapia & -.210 & -.059 & -.168 & -.074 & -.191 & -.256 & -.148 & -.213 & $p \leq .05$ \\
\hline Estado Civil & -0.18 & -0.47 & .056 & .083 & .336 & .106 & .259 & .276 & $\mathrm{p} \leq .05$ \\
\hline Profesión & .190 & -.069 & -.275 & .087 & .016 & -.037 & .068 & -069 & $\mathrm{p} \leq .05$ \\
\hline Nivel de estudios completado & .311 & -.097 & -.127 & .319 & .209 & -.046 & -.176 & .171 & $\mathrm{p} \leq .05$ \\
\hline Religión & -.312 & -.258 & -.269 & -.138 & -.294 & -.049 & -.106 & -.117 & $\mathrm{p} \leq .05$ \\
\hline Personas con las que vive & -.168 & -.199 & .280 & -.207 & -.115 & .043 & -.096 & .038 & $\mathrm{p} \leq .05$ \\
\hline Tiempo desde diagnostico & .381 & .146 & .022 & -.139 & -.058 & .025 & .210 & .071 & $\mathrm{p} \leq .05$ \\
\hline $\begin{array}{l}\text { Consulta con profesional de la } \\
\text { salud (psicólogo/psiquiatra) }\end{array}$ & .039 & -.128 & -.273 & -.356 & -.337 & .033 & -.088 & -.197 & $p \leq .05$ \\
\hline $\begin{array}{l}\text { Tratamiento psicológico- } \\
\text { psiquiátrico }\end{array}$ & -.055 & -.003 & -.479 & -.113 & -.274 & .016 & .037 & -.204 & $\mathrm{p} \leq .05$ \\
\hline
\end{tabular}


Pueden observarse correlaciones estadísticamente significativas entre las variables Radioterapia, Quimioterapia, tiempo transcurrido desde el diagnóstico, fase clínica actual, tipo de cirugía recibida, tratamiento psicológico-psiquiátrico, consulta con profesional de la salud, status laboral, y las escalas del cuestionario.

Al graficar los resultados de los Componentes Físico y Mental en función de la variable clínica Tipo de Cirugía, se vio que las distribuciones se acercan a la normalidad. Por lo tanto, se decidió utilizar la prueba paramétrica ANOVA de comparación de medias, para analizar los Componentes Físico y Mental en función del Tipo de Cirugía recibida.

Al comparar las medias de los Componentes entre los distintos tipos de cirugía, se observó una diferencia significativa en el Componente Mental $(p \leq .05)$. Con respecto al Componente Físico, el valor de $p>.05$, por lo cual la diferencia existente en las puntuaciones en dicho componente entre los diferentes grupos quirúrgicos fue nosignificativa.
Puede decirse entonces, que existe evidencia de que la Calidad de Vida en su Componente Mental fue diferente en por lo menos un tipo de cirugía recibida. Puede observarse a partir de la lectura de las puntuaciones medias, que aquellas pacientes que fueron intervenidas quirúrgicamente mediante una Mastectomía Radical Modificada con reconstrucción mamaria, presentan mejores niveles de Calidad de Vida en todas las dimensiones del MOS-SF-36 V. 2.0.

Se realizó el mismo análisis para comparar los Componentes de acuerdo a lo grupos etáreos.

En relación al grupo etario, no se observaron diferencias significativas. Por lo tanto, dado que $p>.05$, puede decirse que no se tiene evidencia estadística para rechazar la hipótesis nula de que no existe diferencia entre grupos.

\section{DISCUSIÓN}

En relación a los Componentes de Salud Mental y Físico, se observó que las participantes autoinformaron niveles inferiores de Salud Mental que de Salud Física.

Tabla 5

ANOVA. Comparación de medias para el Componente Físico y Mental, según tipo de cirugía recibida

\begin{tabular}{lrrrrr}
\hline & Suma de cuadrados & Gl & Media cuadrática & $F$ & Sig. \\
\hline Componente Físico & 1056.915 & 3 & 352.305 & 2.869 & .053 \\
Inter-grupos & 3684.135 & 30 & 122.805 & & \\
Intra-grupos & 4741.051 & 33 & & & \\
Total & & & & & \\
& & & & & \\
Componente Mental & Suma de cuadrados & Gl & Media cuadrática & $F$ & Sig. \\
& 858.744 & 3 & 286.248 & 3.585 & .025 \\
Inter-grupos & 2395.521 & 30 & 79.851 & & \\
Intra-grupos & 3254.265 & 33 & & & \\
Total & & & & & \\
\hline
\end{tabular}

Tabla 6

ANOVA. Comparación de medias para el Componente Físico y Mental, según grupo etario

\begin{tabular}{lrrrrr}
\hline & Suma de cuadrados & Gl & Media cuadrática & $F$ & Sig. \\
\hline Componente Físico & 229.096 & 2 & 114.548 & .787 & .464 \\
Inter-grupos & 4511.955 & 31 & 145.547 & & \\
Intra-grupos & 4741.051 & 33 & & & \\
Total & & & & & \\
& & & & & \\
Componente Mental & Suma de cuadrados & Gl & Media cuadrática & $F$ & Sig. \\
& 202.803 & 2 & 101.401 & 1.030 & .369 \\
Inter-grupos & 3051.462 & 31 & 98.434 & & \\
Intra-grupos & 3254.265 & 33 & & & \\
Total & & & & & \\
\hline
\end{tabular}


Estos resultados son coincidentes con los documentados por Marques et al. (2005), quienes encontraron que las mujeres sufren con la expectativa de retomar sus actividades normales, tienen dudas respecto a su futuro y temor a la recurrencia de la enfermedad.

En relación a los datos sociodemográficos de las participantes, la mayoría de las mujeres se encontraban casadas $(41,2 \%)$. Este puede ser considerado un factor de protección de la salud, si se tiene en cuenta lo documentado por Shapiro et al. (2001) y Sammarco y Konecny (2008) sobre el impacto positivo que tiene el sostén de la pareja en la CVRS de las mujeres afectadas por una enfermedad crónica.

En lo que refiere al nivel socioeconómico, se debe tener presente que las mujeres estudiadas recibían asistencia médica en el INCA, institución de salud pública del Uruguay, lo cual refleja un nivel socioeconómico medio-bajo a bajo. Este es un factor relevante al pensar en la CVRS de estas mujeres, dado que las investigaciones han documentado que los bajos ingresos están relacionados con peores niveles de Calidad de Vida (NBCC, 2009). Por otro lado, se observó que el $50 \%$ de las participantes había completado sus estudios hasta primaria, lo cual es también un elemento a tener en cuenta, dado que según el NBCC los bajos niveles de educación están asociados con peores niveles de Calidad de Vida. Así mismo, el $26,5 \%$ de las participantes vivían solas. De acuerdo con Schou et al. (2005) este sería otro factor que impacta negativamente en la CVRS de las pacientes.

En relación a la variable edad, un 32,4\% de la muestra se ubicó en el rango etario de 38 a 49 años. El mismo porcentaje de mujeres $(32,4 \%)$ se encontró en el rango de 60 a 78 años de edad. No se observaron diferencias significativas en los niveles de Calidad de Vida entre los diferentes grupos etarios. Esto podría estar vinculado al rango de edad de la muestra, en la cual la paciente más joven tiene 38 años y la de mayor edad 78 años.

El tratamiento más frecuente entre las participantes fue la Quimioterapia (73,5\%), la Radioterapia $(64,7 \%)$ y la combinación de ambos $(58,8 \%)$. De acuerdo con la bibliografía existente, son numerosas las investigaciones que han concluido que el tratamiento médico adyuvante que está relacionado con una mayor disminución en la Calidad de Vida del paciente es la Quimioterapia (p.ej., Hopwood et al., 2007;
Meyerowitz, Sparks, y Spears, 1978). Por otro lado, algunos autores han documentado que el hecho de haber recibido Radioterapia se encontró significativamente asociado a mayor fatiga, y ésta, a peores niveles de Calidad de Vida (p.ej., Graydon, 1994; Jansen et al., 2000). Tanto la Radioterapia como la Quimioterapia son los tratamientos asociados a un mayor impacto en la CVRS de las pacientes.

El tipo de cirugía más frecuente entre las participantes uruguayas fue la Mastectomía Radical Modificada (53\%), seguido por la Cirugía Conservadora (47\%). Las pruebas paramétricas realizadas arrojaron resultados que permiten afirmar que la Calidad de Vida es diferente en por lo menos un tipo de cirugía recibida. Al pensar en la CVRS de estas pacientes, es importante tomar en cuenta que son varios los autores que han documentado que la Mastectomía tiene un mayor impacto en detrimento de la CVRS frente a la Cirugía Conservadora (p.ej., de Haes et al., 2003; Rabin et al., 2008; Wapnir, Cody, y Greco, 1999). Además, es importante tomar en cuenta los hallazgos de Bulotiene, Vesëliunas, y Ostapenko (2007), quienes han encontrado que el empeoramiento de la Calidad de Vida global de las pacientes con Mastectomía y Quimioterapia resulta mayor que el de aquellas mujeres que fueron intervenidas mediante una Cirugía Conservadora o Mastectomía sin Quimioterapia.

En el presente estudio se han hallado variables clínicas y sociodemográficas asociadas significativamente con las diferentes subescalas del MOS-SF-36 V. 2.0.

El tipo de cirugía recibida se encontró asociado significativamente con Vitalidad $(.341 ; p \leq .05)$ y Salud Mental $(.364 ; p \leq .05)$. Las pacientes que han sido intervenidas quirúrgicamente mediante una Mastectomía Radical con reconstrucción mamaria presentan mejores puntuaciones en la escala de Vitalidad y Función Social frente al resto. Estos resultados son similares a los documentados por Bulotienë, Ostapenko, y Vesëliunas (2005) y Girotto, Schreiber, y Nahabedian (2003). Podemos pensar que las mujeres mastectomizadas y posteriormente reconstruidas probablemente se sientan más cómodas socialmente y con una mejor autoimagen corporal frente a aquellas participantes a quienes no se les practicó una reconstrucción mamaria.

Se encontró una asociación significativa y negativa entre Quimioterapia y la dimensión de 
Salud General (-.409; $p \leq .05)$. Es decir, que a mayores dosis de Quimioterapia recibida, menor fue la Calidad de Vida autoinformada por estas mujeres. Resultados similares han sido descritos por Ganz et al., (2002), Hopwood et al., (2007) y Meyerowitz, Sparks, y Spears, (1978). Por otro lado, se encontró que la Radioterapia estuvo asociada significativa y negativamente con Dolor Corporal (-.378; $p \leq .05)$. Aquellas pacientes que recibieron Radioterapia presentan mayor intensidad del dolor en las tareas o trabajos que realizan. Los elevados niveles de Dolor Corporal en las mujeres que recibieron Radioterapia pueden atribuirse a los efectos secundarios del mencionado tratamiento como ser náuseas, fatiga, infecciones, diarreas, entre otros.

La fase clínica actual se encontró asociada significativamente con Rol Emocional (.355; $p \leq .05)$ y Función Física $(-.374 ; p \leq .05)$. Las pacientes que se encontraban en una etapa de remisión parcial o completa puntuaron más alto en Función Física y Rol Emocional en comparación con las que cursaban tratamiento. Esto podría explicarse en función de que una vez que las pacientes se encuentran libres de la enfermedad, pueden desarrollar más cantidad de actividades con menores limitaciones debido a su salud física o emocional, presentando mejores niveles de Calidad de Vida. Por otro lado, es más probable que las pacientes en fases de remisión completa asistan a las consultas médicas con el fin de realizar chequeos periódicos y no estén siendo tan afectadas por los efectos secundarios de los tratamientos adyuvantes.

La ocupación de las pacientes se encontró asociada a la CVRS. El status laboral se encontró correlacionado en forma significativa con Función Física $(-.486 ; p \leq .05)$ y Función Social $(.339 ; p \leq .05)$. Las pacientes laboralmente activas puntuaron más alto en Función Física en comparación con aquellas que no trabajaban al momento de la evaluación. Resultados similares han sido documentados por Safee et al. (2008). Se podría pensar que en tanto la mujer se encuentre trabajando, posiblemente tenga menores dificultades físicas para hacerlo, presente menos limitaciones, y tal vez sea esta la razón que le haya posibilitado trabajar. Por otro lado, las mujeres laboralmente inactivas tienen mejores puntuaciones en la escala Función Social, es decir que pueden llevar adelante actividades sociales sin mayores impedimentos. Podemos pensar que estas mujeres probable- mente cuenten con otras fuentes de apoyo y redes sociales que generen satisfacción a nivel social, así como también con más tiempo de ocio que las mujeres que trabajan.

Además, el tratamiento psicológico-psiquiátrico estuvo correlacionado con la subescala Dolor Corporal $(-.479 ; p \leq .05)$ y haber consultado con un profesional de la salud mental se encontró correlacionado con Salud General (-.356; $p \leq .05)$. En tanto más afectada se vio la Salud General de las participantes, mayores fueron las consultas con profesionales de la salud mental.

Por otro lado, se hallaron asociaciones significativas entre el tipo de cirugía recibida y el tratamiento psicológico-psiquiátrico (-.564; $p \leq .01)$. Las pacientes que fueron intervenidas mediante una Mastectomía fueron quienes mayoritariamente recibieron dicho tratamiento. Este resultado coincide con la literatura revisada sobre el tema y puede comprenderse si consideramos la Mastectomía como el tipo de cirugía que tiene mayor incidencia negativa en la imagen corporal de la mujer, frente a otros tipos de cirugía (p.ej., Janni et al. 2001; Arraras et al., 2004).

No se han encontrado correlaciones significativas entre el estadío de la enfermedad y las subescalas del cuestionario. Se podría pensar que, algunas veces, la no-veracidad o desinformación respecto de ciertas respuestas puede incidir en los resultados de los estudios, perdiéndose de esta manera las posibles correlaciones. Por otro lado, algunos datos no del todo precisos sobre la propia enfermedad podrían estar incidiendo en estos resultados.

Es posible además, que la pequeña muestra empleada en este estudio pueda haber hecho perder poder estadístico y de significatividad. Sumado a ello, el hecho de que el estadío IV de la enfermedad estuviera integrado por una sola participante, no resulta representativo y puede sesgar los resultados.

\section{CONCLUSIONES}

En el presente estudio se evaluó la medida en que la CVRS de las pacientes uruguayas diagnosticadas con Cáncer de Mama en etapas post-cirugía se vio afectada; se buscó establecer la relación existente entre las variables clínicas y sociodemográficas y la CVRS.

Variables como el tipo de cirugía y tratamien- 
tos adyuvantes recibidos, fase clínica actual, status laboral, y tratamiento psicológico-psiquiátrico estuvieron asociadas significativamente a la Calidad de Vida de las mujeres con Cáncer de Mama.

Del análisis de los factores clínicos, se desprende que el tipo de cirugía recibida se encontró asociado a diferencias significativas en la CVRS de estas mujeres. Los resultados permiten concluir que la Mastectomía Radical Modificada con reconstrucción mamaria es la cirugía que tiene un menor impacto en detrimento de las funciones sociales de las participantes, frente a otros tipos de cirugía. El tipo de tratamiento recibido por las participantes también se encontró asociado a la CVRS, reportando niveles de dolor más intensos y mayores impedimentos en sus tareas domésticas o laborales, aquellas mujeres que recibieron Quimioterapia o Radioterapia (p.ej., Graydon, 1994; Jansen et al., 2000; Montazeri, 2008). Consideramos que un manejo efectivo de los efectos secundarios de los tratamientos adyuvantes contribuirá a reducir el impacto de los mismos en la CVRS. Asimismo, posiblemente el intervenir para la reducción de síntomas asociados a la enfermedad y sus tratamientos tenga un efecto positivo en la Calidad de Vida de las pacientes.

La fase clínica en que se encontraba la mujer, también arrojó resultados interesantes a tomar en cuenta en futuras intervenciones psicosociales. Las pacientes que se encontraban en remisión parcial o completa estaban mejor física y emocionalmente que aquellas mujeres que aún cursaban tratamientos adyuvantes. Estos resultados son similares a los hallados por Schou et al., 2005.

En relación a las variables sociodemográficas, dado que no se hallaron diferencias estadísticamente significativas respecto a la edad de las participantes, para futuras investigaciones resultaría interesante retomar estos aspectos, llevando adelante estudios a mayor escala, pudiendo además comparar los resultados obtenidos en mujeres uruguayas saludables.

Como sugerencias para futuras investigaciones, entendemos importante la evaluación de la necesidad de psicoeducación sobre el curso de la enfermedad y los síntomas más frecuentes en períodos post-tratamiento, ya que es éste el momento en que las pacientes mantienen menor contacto con el personal sanitario y aún pueden necesitar apoyo psicosocial.

Por último, es necesario señalar que exis- ten diversas limitaciones en la investigación en el área de la Psicooncología en general, y en Cáncer de Mama en particular. Los hallazgos no concluyentes sobre diversos aspectos que ofrece la literatura relevante en el tema, transforman a la Psicooncología en un área de estudio limitada. En lo que refiere al presente estudio en particular, señalamos que la muestra reducida de participantes podría quitarle poder de detectar relaciones entre variables, o significar que los hallazgos pueden no ser generalizables a poblaciones más amplias o diversas. Sumado a ello, el nivel socioeconómico de la muestra empleada es mayoritariamente bajo, lo cual podría condicionar también su posibilidad de ser generalizados. En cuanto a la enfermedad en sí misma, la muestra estuvo integrada por una sola participante que se encontraba en el estadío IV de la enfermedad; esto puede sesgar los resultados al incluir aquellas pacientes menos severamente enfermas y tal vez mejor ajustadas. Además, los resultados podrían también estar sesgados por el hecho de tratarse de un estudio con muestreo por conveniencia en el cual fueron administrados cuestionarios de autoinforme en la sala de espera previo a la consulta médica, pudiendo ello excluir a aquellas pacientes que se encuentran afectadas en mayor medida, sea física como psicológicamente.

Consideramos importante que las investigaciones futuras tomen en cuentas estas limitaciones, a fin de optimizar la calidad metodológica e interpretación de los resultados que ofrece la base científica actual sobre el tema en nuestro país. Entendemos además que resulta relevante la profundización en los temas mencionados, a fin de contribuir al desarrollo científico de la Psicooncología, con el objetivo primordial de favorecer conductas de protección y promoción de la salud en pacientes oncológicos. Asimismo, resulta de gran relevancia y necesidad para contribuir a la comunidad que futuras investigaciones puedan incluir las fuentes de apoyo social y redes sociales.

\section{REFERENCIAS}

Aaronson, N.K. (1993). Assessment of Quality of Life and benefits from adjuvant therapies in breast cancer. Recent results in Cancer Research, 127, 201-210.

Alonso, J. (1999).Cuestionario de salud SF-36: versión española 1.4. Barcelona: I.M.I.M. 
Antoni, M.H. (2002). Stress Management Intervention for Women with Breast Cancer. Washington D.C.: American Psychological Association Press.

Arraras, J. I., Garrido, E., Pruja, E., Marcos, M., Tejedor, M. y Arias, F. (2000). El cuestionario de Calidad de Vida de la EORTC QLQ-C30 (Versión 2.0). Estudio psicométrico con pacientes con Cáncer de Mama. Clínica y Salud, 11 (3), 329-349.

Arraras, J. I., Illarramendi, J. J., Manterola, A., Salgado, E. y Domínguez, M. A. (2007). Progresos en la evaluación de la Calidad de Vida en el Cáncer de Mama. El sistema de medida de la EORTC. Psicooncología, 4 (2-3), 367-384.

Arraras, J.I., Martínez, M., Manterota, A. y Laínez, N. (2004). La evaluación de la Calidad de Vida del paciente oncológico. El grupo de Calidad de Vida de la EORTC. Psicooncología, 1 (1), 87-98.

Bardwell, W.A., Major, J.M., Rock, C.L., Newman, V.A., Thomson, C.A., Chilton, J.A., et al. (2004). Healthrelated quality of life in women previously treated for early-stage breast cancer. Psycho-Oncology, 13, 595-604.

Barreto, P. y Bayés, R. (1990). El Psicólogo ante el enfermo en situación terminal. Anales de Psicología, 6, 169-180.

Barroilhet, S., Forjaz, M. y Garrido, E. (2005). Conceptos, teorías y factores psicosociales en la adaptación al Cáncer. Actas Españolas de Psiquiatría, 33 (6), 390397.

Bullinger, M. (1991). Quality of Life-definition, conceptualization and implication: a methodologist's view. Theoretical Surgery, 6, 143-149.

Bulotienë, G., Ostapenko, V., y Vesëliunas., J. (2005). Influence of social factors on the quality of life after breast cancer surgical treatment. Acta Medica Lituana, 12(2), 79-83.

Bulotienë, G., Veseliunas, J., y Ostapenko, V. (2007). Quality of life of Lithuanian women with early stage breast cancer. Public Health, 7, 124.

Curran, D., Van Dongen, J., Aaronson, N.K., Kiebert, G., Fentiman, I.S., Mignolet, F. et al. (1998). Quality of Life of early-stage breast cancer patients treated with radical mastectomy or breast conserving procedures: results of the EORTC trial 10801. European Journal of Cancer, 34, 307-314.

de Haes, J. Curran, D., Aaronson, N. y Fentiman, I. (2003). Quality of life in breast cancer patients aged over 70 years, participating in the EORTC10850 randomised clinical trial: On behalf of the EORTC BCCG. European Journal of Cancer, 39 (7), 945-951.

Fossa, S. (1996). New approaches to the treatment of prostate cancer. European Urology, 29 (2), 121-123.

Gaite, L. (2008). Encuentro con expertos. Calidad de vida relacionada con la salud en el niño con enfermedad crónica. Boletín de la Sociedad de Pediatría de Asturias, Cantabria, Castilla y León, 48 (206), 337-346.

Girotto, J.A., Schreiber, J. y Nahabedian, M.Y. (2003). Breast reconstruction in the elderly: preserving excellent quality of life. Annals of Plastic Surgery, 50 (6), 572-578.
Graydon, J. E. (1994). Women with breast cancer: their quality of life following a course of radiation therapy. Journal of Advanced Nursing, 19, 617-622.

Hietanen, P.S. (1996). Measurement and practical aspects of Quality of Life in breast cancer. Acta Oncológica, 35, 39-42.

Hopwood, P., Haviland, J., Mills, J., Sumo, G. y Bliss, J. (2007). The impact of age and clinical factors on quality of life in early breast cancer: An analysis of 2208 women recruited to the UKSTART Trial (Standardization of Breast Radiotherapy Trial). The Breast, 16 (3), 241-25.

Janni, W., Rjosk, D., Dimpfl, T.H., Haertl, K., Strobl, B., Hepp, F. et al. (2001). Quality of life influenced by primary surgical treatment for stage I-III breast cancer-long-term follow-up of a matched-pair analysis. Annals of Surgical Oncology, 8 (6), 542-8.

Jansen, S.J., Stiggelbout, A.M., Nooij, M.A., Noordijk, E.M. y Kievit, J. (2000). Response shift in quality of life measurement in early-stage breast cancer patients undergoing radiotherapy. Quality of Life Research, 9, 603-615.

Lidgren, M., Wilking, N., Jonsson, B. y Rehnberg, C. (2007). Health related quality of life in different states of breast cancer. Quality of Life Research, 16, 1073-1081.

Marques, D., Mendes, A., Cabello, C., Santos-Sá, D., Costa-Paiva, L. y Zangiacomi, E. (2005). Quality of Life in Brazilian Breast Cancer Survivors Age 45-65 Years: Associated Factors. The Breast Journal, 11 (6), 425-432.

Meyerowitz, B., Sparks, f. y Spears, I. (1978). Adjuvant chemotherapy for breast carcinoma: psychosocial implications. Cancer, 43(5), 1613-1618.

Montazeri, A. (2008). Health-related quality of life in breast cancer patients: a bibliographic review of the literature from 1974 to 2007. Journal Of Experimental \& Clinical Cancer Research, 27, 32.

Montazeri, A., Vahdaninia, M., Harirchi, I., Ebrahimi, M., Khaleghi, F. Y Jarvandi, S. (noviembre, 2008). Quality of life in patients with breast cancer before and after diagnosis: an eighteen months follow-up study. Recuperado 10 agosto, 2009, de:http://www.pubmedcentral.nih.gov/ picrender.fcgi?artid=2588619\&blobtype=pdf .

National Breast Cancer Center. (30 de abril, 2009). Recuperado 22 de mayo, 2009, de:http://www.nbocc.org.au/resources/documents/IPD_psychological_distress.pdf.

Osoba, D. (1994). Lessons learned from measuring healthrelated Quality of Life in oncology. Journal of Clinical Oncology, 12, 608-616.

Rabin, E., Heldt, E., Hirakata, V. y Fleck, M. (2008). Quality of life predictors in breast cancer women. European Journal of Oncology Nursing, 12 (1), 53-57.

Radice, D. y Redaelli, A. (2003). Breast Cancer Management. Pharmacoeconomics, 21 (6), 383-396.

Remor, E., Ulla, S. y Arranz, P. (2003). El psicólogo en el ámbito hospitalario. Bilbao: Desclee De Brouwer.

Safee, A., Moghimi-Dehkordi, B., Zeighami, B., Tabatabaee, H.R., y Pourhoseingholi, M.A. (2008). Predictors of quality of life in breast cancer patients under chemotherapy. Indian Journal of Cancer, 45(3), 107-111. 
Sammarco, A. y Konecny, L.M. (2008). Quality of life, social support, and uncertainty among latina breast cancer survivors. Oncology Nursing Forum, 35 (5), 844-849.

Schou, I., Ekeberg, O., Sandvik, L., Hjermstad, M. y Ruland, C. (2005). Multiple predictors of health-related quality of life in early satge breast cancer. Data from a year follow-up study compared with the general population. Quality of Life Research, 14, 1813-1823.

Schwartzmann, L. (2003). Calidad de Vida Relacionada con la Salud: aspectos conceptuales. Ciencia y Enfermería, 9 (2), 9-21.

Shapiro, S., López, A., Schwartz, G., Bootzin, R., Figueredo, A., Braden, C. et al. (2001). Quality of life and Breast Cancer: Relationship to Psychosocial Variables. Journal of Clinical Psychology, 57 (4), 501-519.

Stewart, A.L., Greenfield, S., Hays, R.D., Wells, K., Rogers, W.H., Berry, S.D., et al. (1989). Functional status and well-being of patients with chronic conditions. Journal of the American Medical Association, 262, 907-913.
Stewart, A.L., Hays, R.D., y Ware, J.E. (1988). The MOS ShortForm General Health Survey. Medical Care, 26, 724-735.

Vasallo, J. y Barrios, E. (2003). Actualización Ponderada de los Factores de Riesgo del Cáncer. Montevideo: Comisión Honoraria de Lucha contra el Cáncer. Recuperado 10 de mayo, 2009, de: http://www. urucan. org. uy/ uilayer/ve/ factores_riesgo / factores _ riesgo 26. pdf.

Wapnir, I. L., Cody, R. P., y Greco, R. S. (1999). Subtle differences in quality of life after breast cancer surgery. Annals of Surgical Oncology, 6(4), 359-366.

Ware, J.E. (2000). SF-36 Health Survey Update. Spine, 25, 3130-3139.

Ware J.E. y Sherbourne, C.D. (1992). The MOS 36item short-form health survey. (SF-36): Conceptual framework and item selection. Medical Care, 30, 473-483.

Para citar este artículo:

Bajuk, L. \& Reich, M. (2011). Calidad de vida relacionada con la salud en mujeres uruguayas con cáncer de mama. Ciencias Psicológicas V (1): 19-30. 